\title{
Osteoarticular infections in a tertiary care children's hospital: Epidemiology and clinical characteristics in association with bacteremia
}

\author{
Esmeralda Highton, M.D. ${ }^{a}$, M. Guadalupe Pérez, M.D. ${ }^{a}$, \\ Carola Cedillo Villamagua, M.D.a, María I. Sormani, M.D. ${ }^{a}$, \\ María S. Mussini, M.D. ${ }^{a}$, Adela Isasmendi, Biochemist ${ }^{b}$, José Pinheiro, Technician ${ }^{b}$, \\ Vanesa Reijtman, Biochemist ${ }^{b}$, Moira Taicz, M.D. ${ }^{a}$, \\ Alejandra Mastroianni, Biochemist ${ }^{b}$, María E. García, Biochemist ${ }^{b}$ and \\ María T. Rosanova, M.D. ${ }^{a}$
}

\section{ABSTRACT}

Introduction. Osteoarticular infections are an important cause of morbidity and may present with bacteremia. Theepidemiology has changed in recent years.

Objectives. To describe the epidemiological, clinical, and evolutionary characteristics of children with osteoarticular infections and compare patients with and without bacteremia. Population and methods. Retrospective cohort. Patients younger than 18 years admitted between January 1st, 2016 and December 31st, 2016 suspected of osteoarticular infections who had undergone an arthrocentesis and/or joint biopsy were included. Clinical and laboratory characteristics were compared between patients with and without bacteremia. The Stata 10 software was used.

Results. N: 62. Patients' median age was 59.5 months (interquartile range [IQR]: 24-84). Fever developed in 44 patients $(70 \%)$. Arthritis predominated (54 patients, $87 \%$ ). An etiologic agent was identified in 29 patients $(47 \%)$. Staphylococcus aureus was prevalent (n: 20,32\%). Among these, 15 developed bacteremia (24\%). Clindamycin was administered to 56 patients $(90 \%)$ as empirical therapy. The median intravenous treatment duration was 7 days (IQR: 5-11) and the median length of stay, 7 days (IQR: 4-12). Patients with bacteremia were younger (26 months versus 60 months, $\mathrm{p}<0.05$ ), had a higher baseline C-reactive protein level (101 $\mathrm{U} / \mathrm{L}$ versus $33 \mathrm{U} / \mathrm{L}, \mathrm{p}<0.05)$, a lower hemoglobin level at the time of admission ( $10.8 \mathrm{~g} / \mathrm{dL}$ versus $12.5 \mathrm{~g} / \mathrm{dL}, \mathrm{p}=0.04)$, and a higher frequency of fever $(100 \%$ versus $57 \%, \mathrm{p}<0.05)$.

Conclusions. Staphylococcus aureus was prevalent. Children with bacteremia were younger, had a higher C-reactive protein level, a lower hemoglobin level at the time of admission, and $100 \%$ presented fever.

Key words: osteoarticular infections, child, methicillin-resistant Staphylococcus aureus, bacteremia.

Funding:

None.

Conflict of interest:

None.

Received: 5-19-2017

Accepted: 9-7-2017
To cite: Highton E, Pérez MG , Cedillo Villamagua C, et al. Osteoarticular infections in a tertiary care children's hospital: Epidemiology and clinical characteristics in association with bacteremia. Arch Argent Pediatr 2018;116(2):e204-e209.

\section{INTRODUCTION}

Osteoarticular infections (OAIs) are a diagnostic and treatment challenge for pediatricians. OAIs are a common cause of long-term antibiotic use and hospitalization. They may result in a high morbidity, especially if the initial treatment is inadequate. In general, these are acute infections ${ }^{1}$ that may present with bacteremia in approximately half of cases $^{2}$ and also sepsis. $^{3}$

Beyond the neonatal period, the main etiologic agents are Staphylococcus aureus, Streptococcus pyogenes, and Streptococcus pneumoniae. An increased incidence of community-acquired methicillin-resistant Staphylococcus aureus (CA-MRSA), the use of molecular diagnostic techniques, and the introduction of vaccines in the official immunization schedule have altered the epidemiology of OAIs in the past decades. ${ }^{4}$ Bacteremia in OAIs has been described as a marker of severity. It has been associated with methicillin-resistant Staphylococcus aureus (MRSA) and sepsis. ${ }^{5}$

An early diagnosis of OAI and the initiation of an adequate empirical therapy are essential to reduce subsequent morbidity. An updated knowledge of the epidemiology of this 
type of infections is required to adjust empirical therapy. Even if management is adequate, a significant number of cases may result in irreversible joint damage.

The objective of this study was to describe the epidemiological, clinical, and evolutionary characteristics of children with communityacquired OAIs admitted to a tertiary care children's hospital, and compare the characteristics of patients with and without bacteremia.

\section{POPULATION AND METHODS}

This was a retrospective cohort study. Patients older than 1 month and younger than 18 years, admitted to a tertiary care children's hospital between January $1^{\text {st }}, 2016$ and December $31^{\text {st }}$, 2016, suspected of OAIs, and who had undergone a diagnostic procedure (bone or synovial biopsy and/or arthrocentesis) were included. Children with a history of recent hospitalization, prior osteoarticular pathology and/or immune compromise were excluded.

The medical records of all patients who met the inclusion criteria were reviewed. The medical history, surgery report, and lab tests were recorded.

Age, days of history prior to the visit, clinical presentation, presumptive diagnosis at the time of admission, physical examination, injury location(s), type and number of surgical drains (arthrocentesis, arthrotomy or arthroscopy), days of history at the time of drainage, and length of stay.

Laboratory, joint fluid culture, and blood culture outcome measures, and antimicrobial susceptibility of agents involved were recorded.

The need or not for admission to the intensive care unit, empirical and definite antibiotic therapy -either oral or intravenous- and its duration, treatment response, and development of sequelae within 3 months of follow-up were documented.

Blood culture and joint fluid culture or bone biopsy or synovial biopsy specimens were processed at the hospital's Microbiology Laboratory.

Blood cultures were processed using the automated Bact/Alert 3D system and were then typified using conventional and automated microbiological testing in accordance with the current working protocols established by the Microbiology Laboratory.

Blood agar, chocolate agar, cystine lactose electrolyte deficient (CLDE) agar, and thioglycolate broth were inoculated with the specimens and incubated at $37^{\circ} \mathrm{C}$ for 24 72 hours. Bacterial identification was done using the MALDI-TOF MS (Vitek-MS, bioMérieux). Antibiotic sensitivity tests were done using automated methods (Vitek 2C, bioMérieux) and the disc diffusion method, according to the Clinical and Laboratory Standards Institute (CLSI) Guidelines. ${ }^{6}$

\section{DEFINITIONS}

Empirical therapy: antibiotic therapy indicated before the etiologic agent had been identified.

Definite therapy: antibiotic therapy prescribed once blood and / or joint fluid culture results were available.

Community-acquired infection: infections in patients who had not been hospitalized in the past 6 months and who did not attend a health care center on, at least, a weekly basis.

Antibiotic use in the past week: history of systemic antibiotic use for at least 48 hours (oral or parenteral).

All tests were part of the routine medical care for this disease and in relation to patients' course. Data analysis and publication were done with the consent of patients and their families and protecting their identity. The protocol was approved by the hospital's Research and Ethics Committee.

Statistical analysis: Continuous outcome measures were summarized into median and interquartile range (IQR), and categorical outcome measures, into frequency and percentage. Clinical and laboratory characteristics at the time of admission were compared between patients with and without bacteremia. A bivariate analysis was performed. The rank-sum test was used for continuous outcome measures with an abnormal distribution and the $\chi^{2}$ test was used for categorical outcome measures to compare both groups. A value of $p<0.05$ was considered statistically significant. The Stata 10 software was used.

\section{RESULTS}

Sixty-two patients with OAIs who met the inclusion criteria were included. Of these, 34 $(55 \%)$ were boys. Patients' median age was 59.5 months (IQR: 24-84).

The median number of days prior to the visit was 3 (IQR: 1-6). Before hospitalization, 44 patients $(70 \%)$ had fever. The reason for consultation was pain and functional incapacity in 48 patients $(77 \%)$. Four patients $(6 \%)$ reported 
having received an antibiotic in the week prior to hospitalization.

Patients had arthritis (54 patients, 87\%), osteoarthritis (6 patients, $10 \%$ ), and osteomyelitis (n: $2 ; 3 \%$ ) (Table 1).

Infections were located on the knee in 29 patients $(47 \%)$, on the hip in $23(37 \%)$, on the ankle in $7(11 \%)$, on the shoulder in $3(4 \%)$, on the femur in $3(4 \%)$, on the tibia in $1(2 \%)$, and on the fibula in $1(2 \%)$. Only 5 patients $(8 \%)$ had polyarticular involvement.

At the time of admission, the blood count showed that patients had a median of 13000 white blood cells (IQR: 10 400-16 600). The median baseline C-reactive protein (CRP) level was 47 U/L (IQR: 19-100), and the median erythrocyte sedimentation rate was $71 \mathrm{~mm} / \mathrm{h}$ (IQR: 44-93).
Blood culture and joint culture or bone biopsy were done in all patients included in the study. An etiologic agent was identified in 25 patients (40\%). Staphylococcus aureus was prevalent (n: 20, $32 \%$ ) (Table 2).

Bacteremia was detected in 15 patients $(24 \%)$. The bacteria identified in blood cultures were MRSA (n: 9; 14\%), methicillin-susceptible Staphylococcus aureus (n: $4 ; 6 \%$ ), Neisseria meningitidis (n: $1 ; 2 \%$ ), and Streptococcus pyogenes (n: $1 ; 2 \%$ ).

Empirical therapy was clindamycin $(30 \mathrm{mg} /$ $\mathrm{kg}$ / day) in 56 patients (90\%), vancomycin (40 mg/ $\mathrm{kg} /$ day) in $3(5 \%)$, and ceftriaxone $(50 \mathrm{mg} /$ $\mathrm{kg} /$ day) in $2(3 \%)$. No empirical therapy was indicated in 1 patient because his condition was considered transient synovitis. The intravenous

TABLE 1. Clinical and laboratory characteristics recorded at the time of admission in children with osteoarticular infection with and without bacteremia

\begin{tabular}{lccc}
\hline Characteristic & $\begin{array}{c}\text { Children with bacteremia } \\
\text { (n: 15) } \mathbf{~ \%}\end{array}$ & $\begin{array}{c}\text { Children without bacteremia } \\
\text { (n: 47) N \% }\end{array}$ & P \\
\hline Male sex & $8(53)$ & $26(55)$ & 0.9 \\
Age in months (median, IQR) & $26(12-90)$ & $60(24-85)$ & $0.02^{*}$ \\
Days of history prior to the visit (median, IQR) & $3(1-5)$ & $3(1-7)$ & 0.6 \\
Prior antibiotic use & - & $4(9)$ & - \\
White blood cell count $\left(/ \mathrm{mm}^{3}\right)($ median, IQR) & $13100(9400-17700)$ & $13000(11000-16000)$ & 0.8 \\
Hemoglobin $(\mathrm{g} / \mathrm{dL})$ & $10.8(9.9-12)$ & $12.5(10-13)$ & $0.04^{*}$ \\
Platelet count $\left(/ \mathrm{mm}^{3}\right)($ median, IQR) & $281000(154000-346000)$ & $340000(288000-341000)$ & 0.07 \\
Erythrocyte sedimentation rate $(\mathrm{mm} / \mathrm{h})($ median, IQR) & $65(45-83)$ & $73(43-95)$ & 0.7 \\
C-reactive protein $(\mathrm{U} / \mathrm{L})($ median, IQR) & $101(57-203)$ & $33(17-56)$ & $0.01^{*}$ \\
Fever & $100(15)$ & $57(28)$ & $<0.01^{*}$ \\
Final diagnosis: $\quad$ Arthritis $\quad 12(80)$ & $42(89)$ & 0.6 \\
$\quad$ Osteoarthritis & $3(20)$ & $3(6)$ & $0.03^{*}$ \\
$\quad$ Osteomyelitis & $0(0)$ & $2(4)$ & - \\
\hline
\end{tabular}

* Statistically significant.

IQR: interquartile range.

TABLE 2. Microbiological identification by site of documentation. N: 25

\begin{tabular}{|c|c|c|}
\hline Site of identification & Microorganism & $\mathbf{N}(\%)$ \\
\hline Blood cultures N: 5 & $\begin{array}{l}\text { Methicillin-resistant Staphylococcus aureus } \\
\text { Methicillin-susceptible Staphylococcus aureus }\end{array}$ & $\begin{array}{l}4(16) \\
1(4)\end{array}$ \\
\hline Blood culture and joint fluid culture or bone biopsy N: 10 & $\begin{array}{l}\text { Methicillin-resistant Staphylococcus aureus } \\
\text { Methicillin-susceptible Staphylococcus aureus } \\
\text { Neisseria meningitidis } \\
\text { Streptococcus pyogenes }\end{array}$ & $\begin{array}{l}5(20) \\
3(12) \\
1(4) \\
1(4)\end{array}$ \\
\hline Joint fluid N: 9 & $\begin{array}{l}\text { Methicillin-resistant Staphylococcus aureus } \\
\text { Methicillin-susceptible Staphylococcus aureus } \\
\text { Streptococcus pyogenes + methicillin-resistant S. aureus } \\
\text { Haemophilus influenzae } \\
\text { Kingella kingae } \\
\text { Moraxella catarrhalis }\end{array}$ & $\begin{array}{l}3(12) \\
2(8) \\
1(4) \\
1(4) \\
1(4) \\
1(4)\end{array}$ \\
\hline Bone biopsy N: 1 & Methicillin-resistant Staphylococcus aureus & $1(4)$ \\
\hline Joint fluid culture and bone biopsy $\mathrm{N}: 1$ & Pseudomonas aeruginosa & $1(4)$ \\
\hline
\end{tabular}


treatment lasted a median of 7 days (IQR: 5-11). The median length of stay was 7 days (IQR: 4-12). The definite therapy was clindamycin in 38 patients $(61 \%)$, trimethoprim/sulfamethoxazole in $11(18 \%)$, cephalexin in $6(10 \%)$, amoxicillin in $3(5 \%)$, and ciprofloxacin in $1(2 \%)$. Oral combined therapy with amoxicillin and trimethoprim/ sulfamethoxazole was indicated in 2 patients (4\%). The entire therapy (oral and parenteral) lasted a median of 30 days (IQR: 20-42). Only 1 patient $(2 \%)$ had a complication observed at the 3 -month assessment (pathological fracture).

The characteristics of patients with and without bacteremia were compared. The bivariate analysis (Table 1) identified that children with positive blood cultures were younger ( 26 versus 60 months old, $\mathrm{p}<0.05)$, had a higher baseline CRP level (101 U/L versus $33 \mathrm{U} / \mathrm{L}, \mathrm{p}<0.05)$, a higher hemoglobin level $(10.8 \mathrm{~g} / \mathrm{dL}$ versus $12.5 \mathrm{~g} / \mathrm{dL}, \mathrm{p}=0.04)$, and a higher frequency of fever $(100 \%$ versus $57 \%, p<0.05)$. No statistically significant differences were observed in the bivariate analysis between both groups in terms of sex, days of history prior to the visit, erythrocyte sedimentation rate, white blood cell count, hemoglobin level, and platelet count at the time of admission.

No patient died or required admission to the intensive care unit.

\section{DISCUSSION}

OAIs are severe infections that require adequate and early management to improve prognosis. Since the universal use of conjugate vaccines was introduced in children younger than 2 years and the incidence of CA-MRSA increased, a change in the epidemiology of OAIs has been reported in pediatrics worldwide. ${ }^{7}$

The median age at the time of OAI onset reported in the bibliography ranges from 36 months $^{8}$ to 7 years, ${ }^{5}$ and patients with MRSA infections were older, as in the study described here.

The most common presentation is acute monoarthritis, although polyarticular involvement has been reported in up to $22 \%$ of cases. ${ }^{9}$ Only $8 \%$ of patients in this cohort had polyarticular involvement.

Larger joints are more commonly affected than smaller ones. Consistent with the findings of our series, the most common localizations in children are the hip and the knee. ${ }^{10}$

In a systematic review by Margaretten et al., ${ }^{11}$ the diagnostic usefulness of clinical characteristics for bacterial OAI diagnosis was assessed. Fever, blood count results, erythrocyte sedimentation rate, and CRP level are not enough to rule out a bacterial infection diagnosis. In our study, CRP was statistically associated with positive blood cultures.

The key for diagnosis in the cases of clinically suspected OAIs are microbiological and imaging tests. The microorganism was identified in $50-80 \%$ of patients. ${ }^{12}$ Gram staining of synovial fluid and microscopy show a positive result in only $50 \%$ of septic arthritis cases. ${ }^{13}$ Blood cultures are positive in $60 \%$ of acute cases. In our cohort, an etiologic agent was identified in $40 \%$ of cases.

The prevalent pathogenic microorganisms in children with OAIs are Staphylococcus aureus and Streptococcus pyogenes. ${ }^{14}$ Before the introduction of the Haemophilus influenzae type b conjugated vaccine, OAIs accounted for a presentation form of invasive infections by this Gram-negative bacillus. However, as of the internationalization of the conjugated vaccine, the incidence of this type of infections reduced radically. ${ }^{15}$

Nevertheless, the big change in OAI epidemiology has been the emergence of CAMRSA. In the past decades, MRSA has been broadly prevalent in OAIs in Argentina, which is consistent with the findings of this study. ${ }^{16}$

The surgical management and an early antibiotic therapy improve the course of patients with acute OAIs. In our setting, the fact that MRSA is broadly prevalent in this type of infections should be considered when selecting an empirical antibiotic. ${ }^{17}$

Different studies have assessed differential characteristics of children with OAIs with and without bacteremia. ${ }^{18,19}$ In a study conducted in Finland in patients with OAIs and isolation of methicillin-susceptible Staphylococcus aureus, CRP was the only significantly higher marker in children with positive blood cultures. As in the study, no differences were observed in erythrocyte sedimentation rate or white blood cell count levels.

Another study ${ }^{20}$ conducted in a population with a high prevalence of methicillin resistance reported a greater fever duration, bacteremia, extended length of stay, and more surgical procedures in children with OAIs and MRSA bacteremia. Categorizing patients who have a higher risk for bacteremia helps to identify children who will require a longer length of stay in an early manner and propose early and adequate surgical drains. 
In the bibliography, up to $20 \%$ of children were reported to have sequelae one year later. ${ }^{21}$ A greater number of days elapsed before the visit, a delayed treatment, increased levels of acute phase reactants, ${ }^{22}$ a younger age, and MRSA identification have been described as factors of poor prognosis..$^{23}$ This study was not designed to assess long-term morbidity caused by OAIs in children, so follow-up did not last long enough to study sequelae and draw definite conclusions.

Few studies have been carried out in Argentina on OAIs in children. One of the main strengths of this study is the substantial number of patients suspected of OAIs included in a short period and in only one site. In all cases, patients were assessed in a systematic manner using blood and synovial fluid cultures. Patients with a history of recent hospitalization were excluded to improve the external validity of findings.

However, it is worth noting that the research setting of this study was a tertiary care children's hospital where patients from all over the country are referred. These patients usually require specific diagnostic and therapeutic procedures that are not available in other health care facilities. These characteristics should be taken into consideration before generalizing the findings of this study.

Future studies should include a higher number of patients and explore clinical and laboratory outcome measures in a multivariate analysis.

\section{CONCLUSIONS}

In this cohort of children with OAIs, MRSA was identified as the main etiologic agent. Children with bacteremia were younger, had a higher CRP level, and more frequently developed fever than those with negative blood cultures.

\section{REFERENCES}

1. Paganini HR. Artritis séptica. In: Infectología pediátrica. Buenos Aires: Científica interamericana;2007.Págs.565-75.

2. Rosanova MT.Infecciones osteoarticulares: Artritis sépticas, Osteomielitis. In: Bruno M, Marcó del Pont J, Ellis A, et al. Libro Azul Infectología Pediátrica. 3. ${ }^{\text {ra }}$ ed. Buenos Aires: FUNDASAP; 2007.Págs.178-86.

3. Rosanova MT. Infecciones osteoarticulares. Artritis séptica. Med Infant. 2008;15(4):342-6.

4. Hernández T, Zarzoso S, Navarro M, et al. Osteomielitis y artritis séptica. Protocolos diagnóstico-terapéuticos de la AEP: Infectología pediátrica. Madrid: Hospital Materno-Infantil Gregorio Marañón; 2010. [Accessed on: April 30th, 2017]. Available at: http: / / www.aeped.es/sites / default/files / documentos / osteomielitis.pdf.

5. McNeil JC, Kaplan SL, Vallejo JC. The Influence of the Route of Antibiotic Administration, Methicillin Susceptibility, Vancomycin Duration and Serum Trough Concentration on Outcomes of Pediatric Staphylococcus aureus Bacteremic Osteoarticular Infection. Pediatr Infect Dis J 2017;36(6):572-7.

6. Clinical and Laboratory Standards Institute. Performance Standards for Antimicrobial Susceptibility Testing; TwentyFifth InformationalSupplement. CLSI document M100-S25. Wayne, PA: CLSI; 2015.

7. ArnoldSR, Elias D, Buckingham SC, etal. Changing patterns of acute hematogenous osteomyelitis and septic arthritis: emergence of community-associated methicillin-resistant Staphylococcus aureus. J Pediatr Orthop 2006;26(6):703-8.

8. Bologna R. Tratamiento oral de infecciones severas. Med Infant. 1996;3(3):170-4.

9. Dubost JJ, Fis I, Denis P, et al. Polyarticular septic arthritis. Medicine (Baltimore) 1993;72(5):296-310.

10. Pääkkönen $M$, Peltola $H$. Treatment of acute septic arthritis. Pediatr Infect Dis J 2013;32(6):684-5.

11. Li SF, Cassidy C, Chang C, et al. Diagnostic utility of laboratory tests in septic arthritis. Emerg Med J 2007; 24(2):75-7.

12. Mathews CJ, Coakley G. Septic arthritis: current diagnostic and therapeutic algorithm. Current Opin Reumatol 2008;20(4):457-62.

13. Mathews CJ, Kingsley G, Field M, et al. Management of septic arthritis: a systematic review. Ann Rheum Dis 2007;66(4):440-5.

14. Gupta MN, Sturrock RD, Field M. A prospective 2-year study of 75 patients with adult-onset septic arthritis. Rheumatology (Oxford) 2001;40(1):24-30.

15. Howard AW, Viskontas D, Sabbagh C. Reduction in osteomyelitis and septic arthritis related to Haemophilus influenzae type B vaccination. J Pediatr Orthop 1999; 19(6):705-9.

16. Rosanova MT, Berberian G, Bologna R, et al. Estudio descriptivo de infecciones osteo-articulares en niños en tiempos de Staphylococcus aureus resistente a meticilina de la comunidad (SARM-Co). Rev Chil Infectol 2015;32(3):321-5.

17. Rosanova MT, Berberian G, Ruvinsky S, et al. Infecciones por Staphylococcus Aureus Meticilino resistente de la comunidad. Tratamiento basado en la evidencia. Med Infant 2011;18(1):76-8.

18. Pääkkönen M, Kallio MJ, Kallio PE, et al. C-reactive protein versus erythrocyte sedimentation rate, white blood cell count and alkaline phosphatase in diagnosing bacteraemia in bone and joint infections. J Paediatr Child Health 2013;49(3):E189-92.

19. Peltola H, Räsänen JA. Quantative C-reactive protein in relation to erythrocyte sedimentation rate, fever, and duration of antimicrobial therapy in bacteraemic diseases of childhood. J Infect 1982;5:257-67.

20. McNeil J, Hulten K, Mason E, etal. Bacteremic Staphylococcus aureus Osteoarticular Infections: Observations on Treatment and Outcomes in a Population With a High Prevalence of Methicillin-Resistant Staphylococcus aureus (MRSA). Open Forum Infect Dis 2016;3(Suppl 1): 673.

21. Howard-Jones AR, Isaacs D, Gibbons PJ. Twelve-month outcome following septic arthritis in children. J Pediatr Orthop B 2013;22(5):486-90.

22. Yuan $\mathrm{HC}, \mathrm{Wu} \mathrm{KG}, \mathrm{Chen} \mathrm{CJ}$, et al. Characteristics and outcome of septic arthritis in children. J Microbiol Immunol Infect 2006;39(4):342-7.

23. Vander Have KL, Karmazyn B, Verma M, et al. Communityassociated methicillin-resistant Staphylococcus aureus in acute musculoskeletal infection in children: a game changer. J Pediatr Orthop 2009;29(8):927-31. 